\title{
Drug effects in fixed ratio matching to sample'
}

DONALD E. MINTZ, DENNIS J. MOURER, and MICHAEL D. STEIN, The City College of The City University of New York, N.Y.

Pigeons' fixed ratio matching-to-sample performance was examined under varied Librium and d-amphetamine doses. Both the response rate and the distribution of errors within the ratio were affected. Latencies of matching attempts for errors and correct matches differed, and this relationship was influenced by drug level and position of the trial within the ratio.

D-amphetamine (Dexedrine) and chlordiazepoxide hydrochloride (Librium) have been examined largely with regard to their influence on rate characteristics of operant behavior. The present study provides an analysis of their effects on matching-to-sample performance maintained with fixed ratio (FR) reinforcementreinforcement for each $n^{\text {th }}$ correct response. In this procedure, the accuracy of performance, the speed of performance and their interactions are analyzed for a range of doses of the two drugs. METHOD

The Ss were three adult male White Carneaux pigeons run at $80 \%$ of their ad lib feeding weight. All had extensive prior training on matching-to-sample with FR reinforcement.

The apparatus consisted of a three-key operant test chamber similar to that described by Mintz, Mourer, \& Weinberg (1966). This included an array of 10 lights, spaced vertically $1 \mathrm{in.}$ apart, the entire line $1 / 2$ in. to the right of the right-hand edge of the center response key. Reinforcement (3-sec access to grain) was provided for nine correct matches (FR9). A single matching trial began with illumination of the center key, either red or green, each with a 0.5 probability. Response on this key immediately ended its illumination, simultaneous with the illumination of the two side keys, one red, the other green $(0.5$ probability of each combination). Response to the side key of matching color was correct, darkening both side keys and advancing to FR count, followed by illumination of the center key for the next trial after a delay of $350 \mathrm{msec}$. Response to the non-matching color constituted an error and produced a 6.0-sec darkening of the keys before onset of the center-key light for the next trial.

Each FR cycle began with the lowest of the 10 lights illuminated. The next higher light in the array was illuminated with each correct match. Reinforcement corresponded to illumination of the 10th light.

The Ss were run on the Monday, Wednesday and Friday of each week for 125 to 150 reinforcements a session. After stable behavior was observed the drug regimen was introduced. Librium doses of $0.5,1.0,2.0$ and $4.0 \mathrm{mg}$ and d-amphetamine doses of 0.5 , 1.0 and $2.0 \mathrm{mg}$ were provided through intra-muscular injection, each solution $0.5 \mathrm{cc}$. (The weights of all three birds at the time of running were slightly below $0.5 \mathrm{~kg}$.) Each Friday a drug was used. On all other occasions inert placebos were injected. An irregular sequence was employed for three administrations of each dose of each drug. All injections preceded the test sessions by $15 \mathrm{~min}$. Placebo data were taken from 10 days, randomly selected from this phase of the experiment.

\section{RESULTS AND DISCUSSION}

For both drug and placebo data the first 10 FR cycles of a session were excluded from the analysis. With $2 \mathrm{mg}$ of d-amphetamine there was complete suppression of behavior at the start of a session. This sometimes lasted several hours and several sessions with this dose were curtailed, resulting in limited data sampled.

In Fig. 1 the drug effects in overall accuracy of performance are shown. With Librium no systerndtic changes are indicated while one $\mathrm{S}$ shows an increase in errors as a function of d-amphetamine dose.

For all conditions, error frequencies were greatest at the beginning of an FR cycle and systematically decreased across the nine steps of the FR. This aspect of the performance replicated

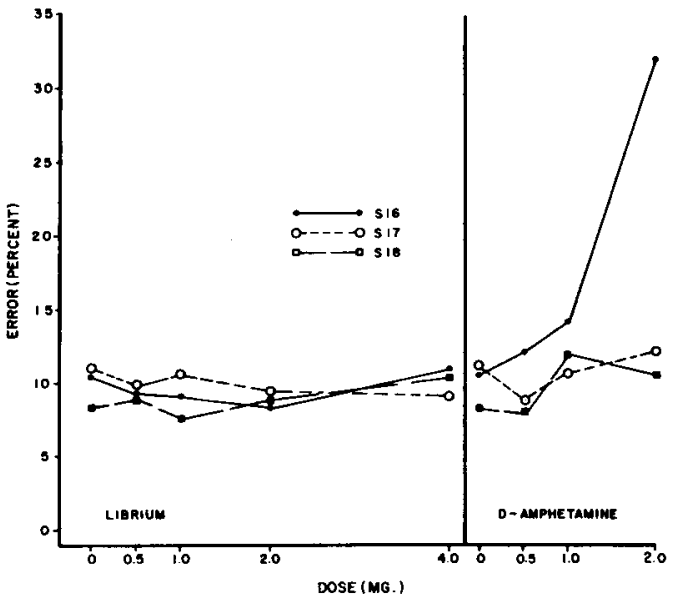

Fig. 1. Matching errors as a function of drug dose.

findings previously reported by Nevin, Cumming, \& Berryman (1963), and Mintz, Mourer, \& Weinberg (1966).

At the lowest doses of Librium $(0.5$ and $1.0 \mathrm{mg})$ the gradients of errors within the FR cycle were nearly identical to those observed under placebo conditions. A tendency toward more frequent error at the first step of the FR was shown for $2.0 \mathrm{mg}$ of Librium. With $4 \mathrm{mg}$ of Librium it was noted that all three Ss showed a sharp rise in the frequency of errors at the first cycle position but a decline in errors later in the cycle, leaving the overall frequency of errors essentially unchanged. Errors in the first cycle position were compared for the placebo and the 4 mg-Librium conditions. Errors rose from $15.5 \%$ to $21.2 \%$ for $\mathrm{S} 16$, from $21.3 \%$ to $29.7 \%$ for S 17 , and from $28.6 \%$ to $40.7 \%$ for $S 18$. D-amphetamine at all three doses produced this type of effect for $S 16$ and $S 18$ but not S 17 .

Both drugs produced changes in matching latency (the time from response on the center key to response on either side key). The medians of matching latency were computed separately for each of the nine cycle positions of the FR. The means of these medians are shown in Fig. 2.

Figure 3 provides the median latency of match attempts, for representative drug doses, plotted separately for errors and correct matches. The placebo data for S 16 show that errors in the first position of the FR cycle correspond to unusually long latencies. However, beyond this position, errors correspond to unusually rapid match attempts. Similar effects can be noted for the placebo performance of S 17 and S 18 (Fig. 4). For S 18 error and correct matching latencies in cycle position 1 show no difference, but

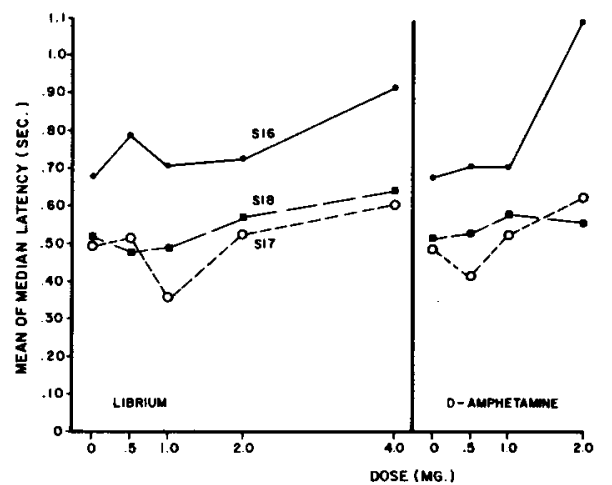

Fig. 2. Latency of match attempt as a function of drug dose. 


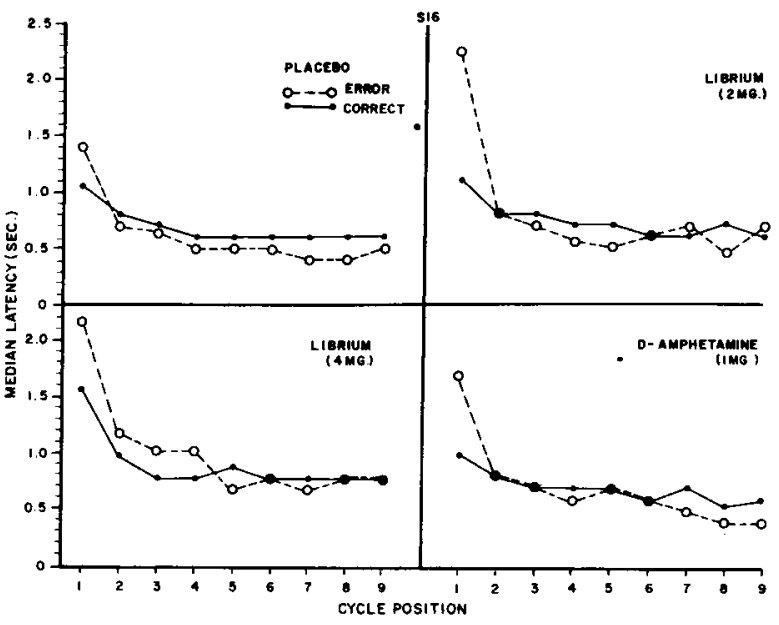

Fig. 3. Latency of error and correct match attempts as a function of FR cycle position for S16. Representative drug doses are shown.

errors in the later positions of the FR cycle are characterized by unusually short latencies.

The effect of both Librium and d-amphetamine was to produce a general increase in latency (Figs. 3 and 4), with errors at the beginning of the FR cycle corresponding to unusually long latencies, even for S 18 (for which this later effect was not shown in the placebo condition). For S 18 there is an indication that the $1 \mathrm{mg} \mathrm{d}$-amphetamine produced long-latency errors throughout the cycle, and indeed a change in the distinctions between latencies

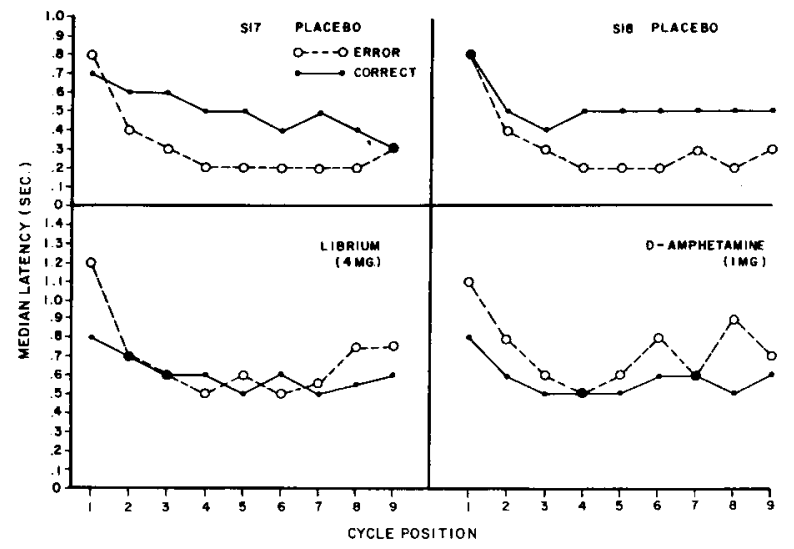

Fig. 4. Latency of error and correct match attempts as a function of FR cycle position for $\mathrm{S17}$ and S18. Representative drug doses are shown. for error and correct was a salient effect of both drugs.

The data suggest that two distinct classes of errors occur in the matching situation. The preponderant class occurs at the beginning of the FR. Here, long latencies before the matching response may generate what is essentially a self-imposed delay. When such delays are experimentally manipulated (Blough, 1959), errors increase with longer delays. The longer latencies in match attempts produced by the drug conditions in the present study may be a determinant of more frequent errors. However, the simultaneous occurrence of an error and a long latency may simply be two independent manifestations of weak control. Nevin, Cumming \& Berryman (1963) also report a correlation between a temporal characteristic of matching performance and probability of error. They found that low response rates on the sample key correlated with poor matching, but in their procedure the sample stimulus (center key) remained illuminated until a match-attempt was made. Thus, changes in the temporal properties of the performance would not constitute a delay unless the effectiveness of the sample stimulus is in some sense limited to when response is made to that stimulus.

In the final steps of the FR the second class of errors is suggested. These, corresponding to unusually short latencies, may reflect control of the response by stimulus factors irrelevant to "correctness." Simple proximity to one key, or head motions initiated prior to side-key onset may be components in the error chains of behavior. These errors, which are relatively infrequent, might be categorized as errors of excessive haste-control by the inertia of the response chain more than the exteroceptive cue. The slowing of performance by the drugs effectively reduced errors of this sort.

Both the drugs and the position of the trial in the FR sequence contribute to the stimulus control as reflected by match-attempt latency. Where control is already weak (the initial FR position) the further weakening by the drugs debilitates performance. However, within the range of Librium employed, there is a facilitation of matching accuracy later in the FR cycle. Although similar effects are shown for d-amphetamine, the higher doses appear to produce sufficiently long latencies that errors of the weak-control variety predominate and an overall increase in error is shown.

\section{REFERENCES}

BLOUGH, D. S. Delayed matching in the pigeon. Journal of the Experimental Analysis of Behavior, 1959, 2, 151-160.

MINTZ, D. E., MOURER, D. J., \& WEINBERG, LAURA S. Stimulus control fixed ratio matching to sample. Journal of the Experimental Analysis of Behavior, 1966, 9, 627-630.

NEVIN, J. A., CUMMING, W. W., \& BERRYMAN, R. Ratio reinforcement of matching behavior. Journal of the Experimental Analysis of Behavior. $1963,6,149-154$.

\section{NOTE}

1. The research was supported in part by grants from Hoffman-La Roche Inc. and the Graduate Division of the City University of New York. 